\title{
Algebraically growing waves in ducts with sheared mean flow
}

\author{
Ali Hasan Nayfeh and Demetri P. Telionis
} Department of Engineering Science and Mechanics, Virginia Polytechnic Institute and State University,
Blacksburg, Virginia 24061

(Received 26 March 1973; revised 4 June 1973)

Standing or traveling waves which vary algebraically with the axial distance in uniform ducts with sheared mean velocity profiles are investigated. The results show that such waves are not possible for ducts with uniform cross sections and fully developed mean flows.

Subject Classification: 20.45; 28.60 .

\section{INTRODUCTION}

Recently, the problem of sound propagation through ducts with mean flow has received considerable attention as evidenced by the large number of contributions (see Refs. 1, 2, and 3). To predict the reduction of sound as it travels through a duct with a given length, most of the authors quoted in the above references assume that all acoustic flow quantities can be expressed in terms of the normal modes in the duct as

$$
\begin{aligned}
Q= & \sum_{n=0}^{\infty} A_{n}\left\{q_{n}(y) \exp \left[i\left(k_{n} x-\omega t\right)\right]\right. \\
& \left.+\bar{q}_{n}(y) \exp \left[-i\left(k_{n} x-\omega t\right)\right]\right\} .
\end{aligned}
$$

Here, $Q$ stands for any of the acoustic flow quantities $u$ (axial velocity), $v$ (normal velocity), $p$ (pressure), $x$ is the axial coordinate, $y$ is the normal coordinate, and $t$ is time. The real and imaginary parts of $k_{n}$ represent, respectively, the wavenumber and attenuation rate of the $n$th mode. In writing Eq. 1, the above authors as sumed that the eigenfunctions between the brackets in Eq. 1 form a complete set.

In this paper we present an indirect argument that these eigenfunctions are complete by showing that (a) there cannot exist standing wave solutions of the acoustic equations of the form

$$
Q=q(x, y) e^{i \omega t}+\bar{q}(x, y) e^{-i \omega t},
$$

where $q(x, y)$ is an algebraic function of $x$, and (b) whereas Nayfeh and Telionis ${ }^{4}$ showed that algebraically varying traveling modes [i.e., $A_{n}=A_{n}(x)$ in Eq. 1] are possible in ducts with varying cross sections, such modes do not exist in uniform ducts.

\section{STANDING WAVES}

The differential equations that govern the propagation of sound in ducts with parallel generators were derived by Pridmore-Brown ${ }^{5}$ from the Euler equations. For a two-dimensional flow with the $x$ axis directed along the axis of the duct, these equations may be expressed in terms of the acoustic pressure $p$ and the normal component $v$ of the acoustic velocity as

$$
\begin{aligned}
\left(1-M^{2}\right) \frac{\partial^{2} p}{\partial x^{2}} & +\frac{\partial^{2} p}{\partial y^{2}}-\frac{2 M}{c} \frac{\partial^{2} p}{\partial x \partial t} \\
& +2 \rho_{0} c \frac{d M}{d y} \frac{\partial v}{\partial x}-\frac{1}{c^{2}} \frac{\partial^{2} p}{\partial t^{2}}=0,
\end{aligned}
$$

$$
\frac{\partial v}{\partial t}+M c \frac{\partial v}{\partial x}+\frac{1}{\rho_{0}} \frac{\partial p}{\partial y}=0
$$

where $\rho_{0}$ is the density of the mean flow, $c$ is the speed of sound, and $M$ is the Mach number which is assumed to be a function of $y$ only. These equations are valid only when the neglected viscous acoustic terms are small compared with retained invisid acoustic terms. Thus Eqs. 3 and 4 are not valid near the boundaries where acoustic boundary layers exist ${ }^{6}$ and near critical layers ${ }^{7}$ where the speed of propagation equals the fluid speed. None of the existing literature of the acoustic propagation in ducts with sheared mean flow takes critical layers into consideration. In this paper, we assume the mean flow speed to be small enough so that critical layers do not exist in the ducts under consideration. For lined walls the boundary conditions are

$$
v / p= \pm \beta / \rho_{0} c \text { at } y= \pm d,
$$

where $\beta$ is the acoustic admittance of the walls and $2 d$ is the width of the duct.

In the farfield we seek asymptotic solutions to Eqs. 3 and 4 in the form

$$
\begin{aligned}
& p=e^{i \omega t} x^{r}\left[F_{0}(y)+x^{-1} F_{1}(y)+x^{-2} F_{2}(y)+\cdots\right], \\
& v=e^{i \omega t} x^{r}\left[G_{0}(y)+x^{-1} G_{1}(y)+x^{-2} G_{2}(y)+\cdots\right],
\end{aligned}
$$

where $r$ is any real number. We substitute Expansions 6 and 7 into Eqs. 3 and 4, equate coefficients of like powers of $x$ to zero, and obtain

$$
\begin{aligned}
& F_{0}^{\prime \prime}+\left(\omega^{2} / c^{2}\right) F_{0}=0, \\
& F_{0}^{\prime}+i \rho_{0} \omega G_{0}=0 ; \\
& F_{1}^{\prime \prime}+\left(\omega^{2} / c^{2}\right) F_{1}=2 i \omega r \frac{M}{c} F_{0}-2 \rho_{0} c r \frac{d M}{d y} G_{0}, \\
& F_{1}^{\prime}+i \rho_{0} \omega G_{1}=-\rho_{0} r M_{c} G_{0} ; \\
& F_{m}^{\prime \prime}+\left(\omega^{2} / c^{2}\right) F_{m}=\xi_{m}, \text { for } m \geqslant 2, \\
& F_{m}^{\prime}+i \rho_{0} \omega G_{m}=\zeta_{m}, \quad \text { for } m \geqslant 2,
\end{aligned}
$$

where $\xi_{m}$ and $\zeta_{m}$ are known functions of $F_{0}, F_{1}, \ldots$, $F_{m-1}$ and $G_{0}, G_{1}, \ldots, G_{m}$.

Substituting Expansions 6 and 7 into Eq. 5 and again equating coefficients of like powers of $x$, we obtain

$$
G_{m}(d) / F_{m}(d)=\beta / \rho_{0} c, \quad m=0,1,2, \ldots .
$$

The symmetric solution of Eqs. 8 and 9 subject to the 
Boundary Condition 14 with $m=0$ is

$$
\begin{aligned}
& F_{0}=A_{n} \cos (\omega y / c), \\
& G_{0}=-\left(i A_{n} / \rho_{0} c\right) \sin (\omega y / c),
\end{aligned}
$$

provided that

$$
\tan (\omega d / c)=i \beta \text {. }
$$

The antisymmetric counterpart of Eqs. 15 and 16 is

$$
\begin{aligned}
& F_{0}=A_{n} \sin (\omega y / c), \\
& G_{0}=\left(i A_{n} / \rho_{0} c\right) \cos (\omega y / c), \\
& \cot (\omega d / c)=-i \beta .
\end{aligned}
$$

Equations 16 and 18 can be satisfied only when $\beta$ is purely imaginary; that is, only when the resistance of the lining material is zero. Lining materials with such a property, though, are considered unrealistic. Therefore, we conclude that standing waves which vary algebraically with the axial distance may be possible only for hard-walled ducts, provided that the frequency is an element of the infinite discrete set

$$
\begin{array}{ll}
\omega_{n}=n \pi c / d, & \text { for symmetric modes, } \\
\omega_{n}=\left(n+\frac{1}{2}\right) \pi c / d, & \text { for antisymmetric modes, }
\end{array}
$$

With $\beta=0$, an inspection of Eqs. $4-7$ reveals that the Boundary Conditions 14 reduce to

$$
F_{m}^{\prime}(d)=0, \quad m=0,1,2, \ldots \text {. }
$$

Taking $\omega=\omega_{n}=n \pi c / d$, we can write Eq. 10 as

$$
\begin{aligned}
F_{1}^{\prime \prime} & +\frac{n^{2} \pi^{2}}{d^{2}} F_{1} \\
& =2 i r A_{n}\left[\frac{n \pi}{d} M \cos \frac{n \pi y}{d}+\frac{d M}{d y} \sin \frac{n \pi y}{d}\right] .
\end{aligned}
$$

Equation 21 has a solution if, and only if, its nonhomogeneous part is orthogonal to the solution of the adjoint homogeneous problem ${ }^{8} \cos (n \pi y / d)$; that is, if, and only if,

$$
\frac{n \pi}{d} \int_{0}^{d} M(y) \cos ^{2} \frac{n \pi y}{d} d y+\frac{1}{2} \int_{0}^{d} \frac{d M}{d y} \sin \frac{2 n \pi y}{d} d y=0 .
$$

Integrating by parts, we can rewrite Eq. 22. as

$$
\dot{S} \equiv \int_{0}^{d} M(y) d y-\int_{0}^{d} M(y) \cos \frac{2 n \pi y}{d} d y=0 .
$$

The case $n=0$ corresponds to a time independent solution which is not of interest. For $n \geqslant 1$, we assume that the mean Mach number profile is uniform and equal to $M_{0}$ in the core of the duct, and possesses a boundary layer profile $M(y)$ in a thin layer of thickness $\delta$ next to the walls. Then

$$
\begin{aligned}
S \equiv & M_{0}(d-\delta)+\int_{d-6}^{\sigma} M(y) d y-M_{0} \frac{d}{2 n \pi} \sin \frac{2 n \pi(d-\delta)}{d} \\
& -\int_{d-6}^{\delta} M \cos \frac{2 n \pi y}{d} d y .
\end{aligned}
$$

A lower bound on $S$ is given by

$$
\frac{S}{M_{0} d}>1-\frac{\delta}{d}-\frac{1}{2 n \pi} \text {. }
$$

As long as $\delta / d$ is less than 0.85 , which is a value very much larger than those encountered in practical applications, $S>0$. Therefore, there cannot exist standing waves that vary algebraically with the axial distance; this conclusion appears to be different from that reached by Möhring. ${ }^{9}$

\section{TRAVELING WAVES}

Next let us determine if traveling waves that vary algebraically along the duct can exist. We seek expansions in the farfield of the form

$$
\begin{aligned}
& p=e^{i \theta} x^{r}\left[F_{0}(y)+x^{-1} F_{1}(y)+\therefore\right], \\
& v=e^{i \theta} x^{r}\left[G_{0}(y)+x^{-1} G_{1}(y)+\cdots\right],
\end{aligned}
$$

where $\theta=k x-\omega t$. Substituting Expansions 26 and 27 into Eqs. 3 and 4 and equating coefficients of like powers of $x$, we obtain

$F_{0}^{\prime \prime}+\left[\frac{\omega^{2}}{c^{2}}-k^{2}\left(1-M^{2}\right)+2 M \frac{\omega}{c} k\right] F_{0}+2 i \rho_{0} c k \cdot \frac{d M}{d y} G_{0}=0$,

$F_{0}^{\prime}+i \rho_{0}(\omega-k M c) G_{0}=0$,

$F_{1}^{\prime \prime}+\left[\frac{\omega^{2}}{c^{2}}-k^{2}\left(1-M^{2}\right)+2 M \frac{\omega}{c} k\right] F_{1}+2 \rho_{0} c \frac{d M}{d y} i k G$

$$
=\frac{2 M i \omega}{c} r F_{0}-2 \rho_{0} c r \frac{d M}{d y} G_{0}-2\left(1-M^{2}\right) i k r F_{0},
$$

$F_{1}^{\prime}+i \rho_{0}(\omega-k M c) G_{1}=-M c r G_{0}$

$F_{m}^{\prime}+\left[\frac{\omega^{2}}{c^{2}}-k^{2}\left(1-M^{2}\right)+2 M \frac{\omega}{c} k\right] F_{m}+2 \rho_{0} c \frac{d M}{d y} i k G=\xi_{m}$,

for $m=2,3, \ldots$,

$F_{m}^{\prime}+i \rho_{0}(\omega-k M c) G_{m}=\zeta_{m}$

For a mean velocity profile that vanishes at the walls, the Boundary Conditions 14 still hold.

Eliminating $G_{0}$ from Eqs. 28 and 29 we have

$$
\begin{aligned}
L\left(F_{0}\right) \equiv & F_{0}^{\prime \prime}-\frac{2 k c}{\omega-M k c} \frac{d M}{d y} F_{0}^{\prime} \\
& +\left[\frac{\omega^{2}}{c^{2}}-k^{2}\left(1-M^{2}\right)+2 M \frac{\omega}{c} k\right] F_{0}=0 .
\end{aligned}
$$

Note that Eq. 34 has a singularity at $y_{c}$ where $M\left(y_{c}\right)$ $=\omega / k c$; a critical layer exists near such a singularity and the acoustic viscous terms cannot be neglected in such a layer. In what follows, we assume the mean Mach number flow to be small enough so that $M<\omega / k c$ everywhere in the duct. For each frequency there exists an infinite number of eigenvalues $k$ and eigenfunctions $F_{0}$ that satisfy Eq. 34, the Boundary Condition 14, and $F_{0}(0)=0$ or $F_{0}^{\prime}(0)=0$. These eigenfunctions can be obtained either a nalytically or numerically. In what follows, we consider only the case of distinct eigenvalues.

Eliminating $G_{1}$ from Eqs. 30 and 31 and using Eq. 34, we obtain

$$
L\left(F_{1}\right)=\psi_{1}\left[M(y), F_{0}(y)\right],
$$

where $\psi_{1}$ is a known function of $M(y)$ and $F_{0}(y)$. For a given frequency, the nonhomogeneous first-order problem has a solution if, and only if, its nonhomogeneous 
part is orthogonal to the solution of the adjoint homogeneous problem. This solvability condition imposes a restriction on the mean Mach number profile. Carrying the expansion to higher order, we find more restrictions on $M(y)$. If $r$ is a positive integer, there will be $r$ restrictions on $M(y)$; otherwise, there will be an infinite number of restrictions on $M(y)$. Since $M(y)$ must satisfy the Navier-Stokes equations, it will not satisfy, in general, any other restriction.

\section{RESULTS AND DISCUSSION}

The results show that there cannot exist standing waves which vary algebraically along a uniform duct if the walls have a finite resistance. Since Nayfeh ${ }^{6}$ showed that the acoustic boundary-layer produces an effective finite admittance at the wall even if it is rigid, we conclude that there are no standing waves which vary algebraically along a duct with uniform cross section.

Also the results show that traveling waves which vary algebraically along the duct do not exist unless the mean velocity profile satisfies one or more restrictions besides satisfying the Navier-Stokes equations. Clearly such velocity profiles are not realistic.

It should be mentioned that our analysis is valid only when the flow speed is everywhere less than the speed of propagation. If these speeds are equal anywhere in the duct, the acoustic viscous terms must be included within the so-called critical layer. Neglecting these viscous terms, one may find a continuous spectrum of eigenvalues due to the fact that the invisid equation has a singularity, ${ }^{10}$ and hence, the initial value problem might have algebraically growing traveling waves. ${ }^{11}$

\section{ACKNOWLEDGMENT}

This work was supported by the Loads Division of the NASA Langley Research Center under Grant NGR 47004-109.

${ }^{1}$ J. F. Unruh and W. Eversman, "The Transmission of Sound in an Acoustically treated Rectangular Duct with Boundary Layer," Wichita State Univ. , Aeronautical Rep. , 72-2 (May 1972).

${ }^{2}$ H. H. Hubbard, D. L. Lansing, and H. L. Runyan, "A Review of Rotating Blade Technology," J. Sound Vib. 19, 227-249 (1971).

${ }^{3}$ M. J. Lighthill, "The Fourth Annual Fairey Lecture: The Propagation of Sound Through Moving Fluids, "J. Sound Vib. 24, 482-492 (1972)

${ }^{4}$ A. H. Nayfeh and D. P. Telionis, "Acoustic Propagation in Ducts with Varying Cross Sections," VPI Engineering Rep. VPI-E-73-7 (Mar. 1973).

${ }^{5}$ D. C. Pridmore-Brown, "Sound Propagation in a Fluid F lowing through an Attenuating Duct," J. Fluid Mech. 4, 393-406 (1958).

${ }^{6}$ A. H. Nayfeh, "Effect of the Acoustic Boundary Layer on the Wave Propagation in Ducts," VPI Engineering Rep. VPI-E73-10 (Apr. 1973).

${ }^{7} \mathrm{C}$. C. Lin, The Theory of Hydrodynamic Stability (Cambridge U. P. , Cambridge, England, 1966), Chap. 8.

${ }^{8}$ E. L. Ince, Ordinary Differential Equations (Dover, New York, 1956), pp. 213-214.

${ }^{9}$ W. Möhring, "On the Resolution into Modes of Sound Fields in Ducts with Shear Flow," presented at the Symposium on "Acoustics of Flow Ducts," Institute of Sound and Vibration Research, University of Southampton (Jan, 1972).

${ }^{10} \mathrm{C}$. C. Lin, "Some Mathematical Problems in the Theory of Stability of Parallel Flows," J. Fluid Mech. 10, 430-439 (1971).

${ }^{11}$ K. M. Case, "Stability of Inviscid Plane Couette F low, " Phys. Fluids 3, 143-148 (1960). 